\title{
Immunotherapy Choice Issues in Crohn's Disease and Ulcerative Colitis
}

\author{
VA Voinov* \\ Apheresis Therapy Department, Pavlov First St. Petersburg State Medical University, Russia \\ *Corresponding Author: VA Voinov, Apheresis Therapy Department, Pavlov First St. Petersburg State Medical University, Russia. \\ Received: August 21, 2019; Published: September 01, 2019
}

DOI: 10.31080/ASGIS.2019.02.0075

Both Crohn's disease and ulcerative necrotic colitis are autoimmune diseases involving disorders of both cellular and humoral immunity. There is a well-known specialization of T-helpers producing cytokines. Thus, type I T-helpers (Th-1) mainly affect the cellular immunity (hypersensitivity and cytotoxicity) and produce IL-2, TNF- $\alpha$ and interferon (IFN)- $\beta$. Cells of type Th- 2 affect the humoral immunity (antibody formation) and secrete IL-4, IL-5 and IL-10, activating B-lymphocytes, stimulating organ-specific autoantibodies formation. Their interaction with antigens in the presence of complement leads to formation of circulating immune complexes (CIC) - "antigen+antibody+complement". Penetrating in the tissues, immune complexes contribute to attraction of macrophages, neutrophils and monocytes, eosinophils and lymphocytes in them associated with excitation of their enzymatic activity, and some released biologic active substances (BAS) cause different types of tissue reactions such as aseptic immune inflammation. The serum of these patients contains antibodies to antigens of the colon mucosa, as well as anti-neutrophil cytoplasmic antibodies.

In active ulcerative colitis [UC] resistant to mesalazine, escalation to either steroids or immunosuppression is common practice. They should suppress the activity of both T- and B-lymphocytes; but the formed cytokines and autoantibodies, remain in the body and continue their destructive effect on the tissues and target organs. However, such therapy causes many adverse reactions. Corticosteroids lead to Cushing's syndrome associated with hypertension, diabetes and osteoporosis, which will require additional treatment of these essentially iatrogenic diseases. Cytostatics lead to significant metabolic disorders, including healthy organs and systems.

In recent years, autoimmune diseases treatment using chimeric monoclonal antibodies to CD20-antigen of B-lymphocytes (rituximab et al.) has become widespread, which should reduce the autoantibodies production. However, there are complications of such treatment up to multiple organ failure. Cetuximab, rituximab, and panitumumab have direct nephrotoxic effect. Selective inhibitors of adhesion molecules, which are represented by natalizumab - a recombinant monoclonal antibody, are also considered promising. However, such treatment has a flip side, which is progressive multifocal leukoencephalopathy development.
Leukocytes releasing toxic cytokines also play a significant role in the pathogenesis. That is why special methods of granulocyte/ monocyte apheresis therapy with adsorption of leukocytes using Ad column are suggested. Photopheresis methods are also used for these purposes. But the isolated removal of just lymphocytes is not accompanied by removal of antibodies and other pathological metabolites.

However, taking into account the autoimmune nature of the disease, extracorporeal itmmunopharmacotherapy can be more effective, when centrifugation removes plasma and isolate leukocytes, which are incubated at $37^{\circ} \mathrm{C}$ for three hours with a minimal dose of corticosteroids (up to $8 \mathrm{mg}$ of dexamethasone), and then returned to the patient intravenously. At the same time, within a small volume each lymphocyte is affected by extremely larger dose of corticosteroids (and in some cases of cytostatics) than in pulse therapy with minimal impact on the entire body. That is, there is a "targeted" immunosuppression of only immunocompetent cells, without affecting the whole body. The course of treatment consists of four such procedures performed every other day.

Thus, with this method, not only the suppression of leukocyte activity occurs, but also the removal of autoantibodies and other pathological metabolites, which should provide a more complete therapeutic effect. Such immunosuppression effect is kept for half a year.

\section{Volume 2 Issue 8 October 2019 (C) All rights are reserved by VA Voinov.}

